\begin{tabular}{|lr}
\multicolumn{2}{|c|}{$\begin{array}{c}\text { Criteria for evaluation of success of blended learning } \\
\text { methodology }\end{array}$} \\
$\begin{array}{lr}\text { Betty Collis, Anoush Margaryan } \\
\text { University of Twente }\end{array}$ & Authors \\
\hline
\end{tabular}

\begin{abstract}
The term blended learning can be interpreted in various ways. Typically in the current corporate-learning context, it refers to a course or series of learning activities in which some of the activities are carried out with the support of technology outside of a face-to-face meeting or classroom setting while other portions of the course take place in a face-to-face meeting or classroom setting. However, this is not the only blend that can occur. Blends also involve different combinations of learning resources and activities, of interactions and communication, and of technologies, all of which take on meaning within the organizational and personal cultures in which the learning occurs. Criteria for the evaluation of blended learning should include measures that capture the context and design of the learning as input variables, measures that capture key aspects of the process of learning as it occurs, and a variety of output measures that capture the impact of the learning. In this paper a particular approach is described which integrates these different sorts of variables through five different sources of data related to a model that relates course input, processes, and outputs. Challenges in carrying out the approach in practice are also discussed.
\end{abstract}

\title{
Evaluation goals and processes
}

The goals of an evaluation of a course in higher education or corporate training can broadly be seen as either obtaining information that can lead to improvement of the course or obtaining information that can help decision makers decide if a course is worth retaining. The first is called formative evaluation, while the latter, referring to the impact of the course, is called summative evaluation. In practice, these distinctions often overlap. The goals of an evaluation can vary among the different stakeholders involved: for the course designer and instructor the focus will emphasize the improvement of specific aspects of the course while for the institutional manager or decision maker the focus will be on the overall costs and benefits of a course or more often, a set of courses. Kirkpatrick's four levels of evaluation (1998) are frequently used as a frame of reference in training contexts. These levels are:

1. The participant reaction level (satisfaction)

2. Learning, in terms of what can be measured or estimated in the classroom or learning setting

3. New or changed behaviour or performance on the job

4. Impact of the training on the organization, including cost considerations 
Evaluation at each of these levels can be used to improve the design of a course or to decide on the value and continuation of a course, or both. In practice, in corporate training, the data collected typically focuses on the participant-satisfaction level, as tapped in an end-of-course questionnaire. This way of thinking about course evaluation is not typical in the context of university courses where courses are not associated with work-based performance or organizational productivity and where learning is generally assessed in terms of the work that students submit and their scores on examinations. However, in both corporate and university contexts, formative evaluation of the learning environment and the learning processes should occur. The ways that learning materials and activities contribute to the learning process are particularly important. A special methodology has evolved around the evaluation of computer-based learning resources for higher education (see for example, Phillips, Bain, McNaught, Rice, \& Tripp, 2000). Computer- and network technology can be used not only for content delivery (the so-called "e-modules") but also to support other learning processes, such as communication, social interaction, assignment submission and feedback, and contributions from the learners themselves to each others' learning (Oliver \& Herrington, 2001). Also, the instructional design of the course can be evaluated; Merrill (2002) has indicated five "first principles" for effective instruction that can be used as criteria. These are that "learners should be engaged in solving real-world problems, that existing knowledge should be activated as a foundation for new knowledge, that new knowledge should be demonstrated to the learner, that the learner should apply the new knowledge, and should integrate it into his world" (pp. 44-45). In general, regardless of the context, course evaluation involves variables relating to the individual learner, the learning environment, the learning technology and resources, pedagogy, and fit of the course to the organizational goals and culture (Anderson, 2004).

\section{Evaluation and blended learning}

The term blended learning is not typically used in universities, although blends of different locations for learning are in fact part of any course in higher education. In the corporate context, blended learning typically refers to a course in which some of the learning activities are carried out with the support of technology outside of a face-to-face meeting or classroom setting while other portions of the course take place in a face-to-face meeting or classroom setting. This implies a new stakeholder in the evaluation process: the workplace supervisor of the learner who influences the quality and possibilities of the learning environment for the participant. The workplace supervisor should provide time, computer and network access, and recognition for the non-classroom learning activities that occur, and can also function as a "learning partner" for the transfer of training to workplace activities (Bianco \& Collis, 2004). Thus, evaluation of blended learning in the corporate context should also include indicators related to the quality of the workplace as a learning environment, particularly with respect to supervisor support but also to other aspects of the organizational culture. For technical professionals, such as geoscientists in the oil industry, the workplace as a learning environment takes on special aspects, in that workplace settings are often in physically challenging environments, where finding a time and place for blended learning and computer assess may be difficult to manage.

Combining the general variables mentioned by Anderson with the extra perspective of the workplace environment suggest a model such as that shown in Figure 1 to guide the evaluation of blended learning for technical professionals where workplace learning involving 
network technology and work-based activities reflecting Merrill's first principles form an important part of the course.

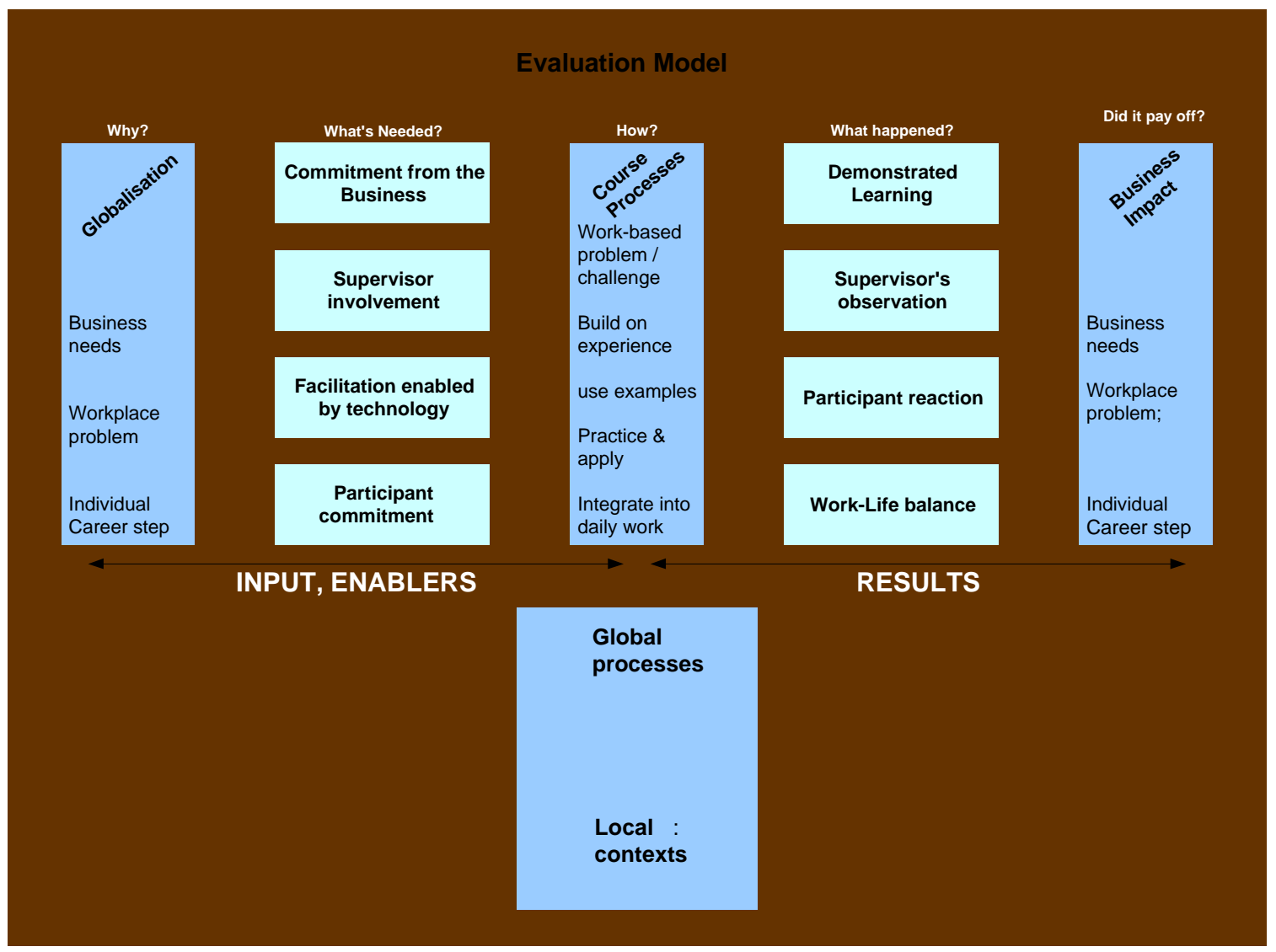

Figure 1. Model for evaluating blended learning for technical professionals (Collis, 2003)

\section{From the Model to Criteria}

Once a model such as that shown in Figure 1 is in place, criteria and procedures for evaluation can be systematically identified. Because of the multi-faceted nature of the model it is clear that one end-of-course participant questionnaire will not be enough to capture the data that will be needed for either formative or summative evaluation. Five different data-capture instruments are needed. These are:

1. A "learning agreement" or contract between the supervisor and participant in which a workplace problem is identified and the performance that the participant should demonstrate to contribute to solving the problem is specified. This document not only specifies the need for the course but serves as the basis for assessing the impact.

2. A "course scan" checklist, in which aspects of instructional design (in particular, Merrill's first principles) and aspects of effective use of technology for the support of learning are applied, and via which the quantity and quality of work-based activities and participant submissions and instructor feedback are tracked.

3. A supervisor's feedback form, in which the workplace supervisor indicates the extent to which he has observed the desired changes in performance. 
4. A participant's feedback form, in which the participant indicates his or her level of satisfaction, perception of learning, usability of the learning technology, perception of the workplace as a learning environment, amount and nature of supervisor support, and amount of sharing and learning with others in the business that occurred in the course.

5. An instructor's reflection form, in which the course instructor reflects on what occurred in the course; the cost in terms of time, energy, and resources; the uses of technology and their effectiveness and efficiency; and in particular, what can be reused from the course, including from participant submissions for subsequent cycles of the course (or for informal learning).

The evaluation methodology now in place for courses offered by the faculty in the Shell EP Learning \& Leadership Development Unit (LLD-SIEP) includes these five sets of input (Nicholson, Masseling, Collis, Margaryan, \& Bianco, 2003). Indicators relating to each of the cells in Figure 1 appear in one or more of these five sets of variables. The course-scan process, for example, includes 61 items that are coded via an after-course analysis of the Web environments used to support all aspects of the blended-learning courses offered by LLDSIEP. Indicators relate to: clarity of the learning objectives and relationship to business needs, design of the learning activities for the course, usability aspects of the course Web environment, and indicators relating to pedagogy such as opportunities for collaboration and for learning with and from others. Indicators also relate to the amount of material used or reused that comes directly from the business, and to the extent to which the supervisor is involved in some aspect of the learning. Also, all courses are moving toward the use of a learning agreement, involving the participant and his or her workplace supervisor (Bianco \& Collis, 2004) and these learning agreements are coded as part of the evaluation. From the learning agreements, indicators are coded that relate to support in terms of time and access to the technology needed for learning, and in terms of the clarity with which specific business needs are identified and related to the plan for work-based activities that the supervisor and participant agree upon for the course. The supervisor's questionnaire asks specifically if the plans made for the course have been carried out, and what impact the course activities are having on the participant's workplace performance. From the instructor's questionnaire, indicators are captured of the time spent on the course, any problems which occurred in terms of participant participation, of reasons for course design decisions and plans for revision, and also of plans for use and reuse of resources submitted by participants or otherwise obtained during the course. From the participant's questionnaire, in addition to questions relating to the participant's subjective reaction to each of the course components and self-appraisal of the current and future value of the different course activities, there are also questions which related to the participant's work-life balance and how the workplace portions of the blended course relate to that balance, and also the participant's impression of the learning climate in his or her workplace situation.

\section{Conclusions}

Having all course submissions and feedback integrated within the same Web environment as the electronic study resources and tools for communication and collaboration means that course processes can be studied and coded. If a course does not have such use of a Web-based environment, there will be difficulty in tracking course processes, particularly in the workplace but also for the classroom component of a blended course. Similarly, if a course 
does not include the use of a learning agreement or a similar device to capture the expectations of the supervisor and participant with respect to performance change, measuring such change will be difficult if not possible to do in a valid and reliable fashion. If a course is to have an impact on the business, it must do this via having an impact on the actual workbased situation of the participants. Thus evaluation is not only monitoring the course-design process, it is also steering it.

\section{References}

Anderson, L. (2004). Gauging the effectiveness of e-learning in education. In P. Resta (Ed.), E-learning for teacher development: A policy and planning guide. Paris: UNESCO. (in press)

Bianco, M., \& Collis, B. (2004). Tools and strategies for engaging the supervisor in technology-support work-based learning, evaluation research. In T. M. Egan, M. L. Morris, \& V. Inbakumar (Eds.), Proceedings AHRD 2004 Conference, Volume 1 (pp. 505-512).

Bowling Green, OH: Academy of Human Resource Development.

Collis, B. (2003). Evaluation- Value to the business. Internal report, Shell EP Learning \& Leadership Development, Noordwijkerhout, NL.

Kirkpatrick, D. (1998). Evaluating training programs: The four levels. $2^{\text {nd }}$ Edition. San Francisco: Berrett-Koehler.

Merrill, D. (2002). First principles of instruction. Educational Technology Research \& Development, 50(3), 43-59.

Nicholson, G., Masseling, I., Collis, B., Margaryan, A., \& Bianco, M. (2003). E-valuation: Learning evaluation system. Internal report, Shell EP Learning \& Leadership Development, Noordwijkerhout, NL.

Oliver, R., \& Herrington, J. (2001). Teaching and learning online. Perth, Australia: Edith Cowan University.

Phillips, R., Bain, J., McNaught, C., Rice, M., \& Tripp, D. (2000). Handbook of learningcentred evaluation of computer-facilitated learning projects in higher education. Melbourne, Australia: Murdoch University. Available online at http://cleo.murdoch.edu.au/projects/cutsd99/ 